\title{
Are Animal Models Useful in Medical Research?
}

\section{Francisco Javier Buils*}

Specialization in General and Digestive Surgery. General Surgery Service and digestive. Sant Joan University Hospital. Rovira and Virgili University, Reus Tarragona, Spain.

Correspondence to: Francisco Javier Buils, Specialization in General and Digestive Surgery, General Surgery Service and Digestive. Sant Joan University Hospital. Rovira and Virgili University, Reus Tarragona, Spain.

Received date: April 2, 2021; Accepted date: April 16, 2021; Published date: April 22, 2021

Citation: Buils FJ (2021) Are Animal Models Useful in Medical Research?. J Med Res Surg 2(2): pp. 1-1. doi: 10.52916/jmrs214044

Copyright: (C)2021 Buils FJ. This is an open-access article distributed under the terms of the Creative Commons Attribution License, which permits unrestricted use, distribution and reproduction in any medium, provided the original author and source are credited.

A nimals, from the pomace fly to the mouse, are widely utilized in research project. They are crucial for allowing scientists to find out more about human biology and health, and for developing new medicines. The use of animals in research project has long been the topic of heated debate. On the one hand it's considered morally wrong to use animals during this way solely for human benefit. On the other hand, our understanding of health and disease would impede after removing animals completely from the lab, and consequently affect the event of latest and vital treatments [1]. No responsible scientist wants to use animals or cause them unnecessary suffering if it are often avoided, and thus scientists accept controls on the utilization of animals in research [2].

Are animal models useful? Animal models are wont to address a spread of scientific questions, from basic science to the event and assessment of novel vaccines, or therapies. The use of animals is not only supported the vast commonalities within the biology of most mammals, but also on the very fact that human diseases often affect other animal species. It is particularly the case for many infectious diseases but also for quite common conditions like Type I diabetes, hypertension, allergies, cancer, epilepsy, myopathies then on. Not only are these diseases shared but the mechanisms are often also so similar that $90 \%$ of the veterinary drugs wont to treat animals are identical or very similar to those used to treat humans. Due to observations and testing on animal models, a number of major breakthroughs in basic science and medical research are possible. Most vaccines, which save many human and animal lives per annum, are successfully developed using animal models. However the results obtained on animals aren't necessarily confirmed in further human studies [3]. No animal model is ever perfect and there are still many differences between model organisms and humans [1].

To help minimise the harm animals may experience while being studied within the laboratory, researchers are required to follow a group of principles, the 'three Rs'. These three Rs Reduction, Refinement, Replacement was proposed by Russel and Birch in 1959 [4]. Replace: Replacing an animal experiment to the best possible extent, as long as adequate alternatives are available. Reduce: The reduction of animal experiments and therefore the number of laboratory animals to the best possible extent. In so doing, it's important to use as many animals as are needed to get a statistically significant outcome. An adequate number of animals are required for the results to be sufficiently significant. Refine: The methods and treatment of the animals during the experiments, and with reference to the way they're kept, should make sure that the distress caused to them is minimized to the best possible extent which their well-being is taken under consideration as far as possible [5].

The inspection and licensing of animal premises, the training and competence of all personal designing projects, performing animal procedures and taking care of animals are the regulatory provisions with which research on animals is conducted and therefore the mandatory authorization of each project by a competent authority upon ethical evaluation by an Animal ethics panel. The criteria for evaluation are supported the 3 Rs rules and a cost-benefit analysis to guage if the potential harm to the animals, which must be reduced to the lowest possible level, is outweighed by significant progress in terms of data on human or animal health. Modern medical research facing the greatest challenges that concern complex, multifactorial, diseases such as cancer, infectious diseases, cardiovascular diseases, neurodegenerative disorders, pathological consequences of aging among others, for which all experimental approaches are indispensable. Research on animal models will remain for an extended time a crucial step for fundamental discoveries, for testing hypotheses at the organism level and for the validation of human data. Likewise, animal protection requires permanent consideration. These two objectives, far away from being antagonistic, must be anchored in high-quality science [3].

\section{References}

1. https://www.yourgenome.org/debates/should-animals-beused-in-research

2. Festing S, Wilkinson R (2007) The ethics of animal research. Talking Point on the use of animals in scientific research. EMBO Rep 8(6): pp. 526-530.

3. Barré-Sinoussi F, Montagutelli X (2015) Animal models are essential to biological research: issues and perspectives. Future Sci OA 1(4): pp. FSO63.

4. Lee $K H$, Lee $D$, Kang BC (2020) The ' $R$ ' principles in laboratory animal experiments. Lab Anim Res 36: pp. 45.

5. https://naturalsciences.ch/animal-experimentation-explain ed/introduction/3rs_principles 